\title{
DIY: How to Help
}

Finally, here are two ways in which you can help. (1) Help us and others build and maintain lists of unsolved problems which can be quickly understood by high school students. (2) Set up a similar program on your own or collaborate with us on a joint project! For example, a Camp Euclid-type program could run (a) in a residential-camp format, (b) in a classroom, (c) at a science festival booth (as shown in Figure 2) or, (d) at the other extreme, online but entirely asynchronously with very low bandwidth requirements.

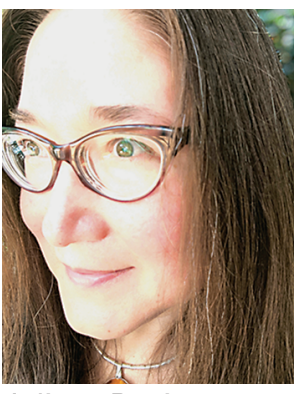

Juliette Benitez

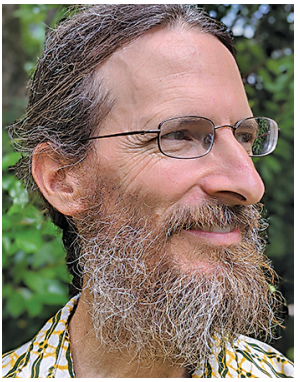

David T. Gay

\section{Credits}

Figure 1 is courtesy of Gregory Araya.

Figure 2 and photo of Juliette Benitez are courtesy of Juliette Benitez.

Photo of Nickolas A. Castro is courtesy of Nickolas A. Castro. Photo of David T. Gay is courtesy of David T. Gay.

\section{Math SWAGGER: Building a Virtual Community}

Student Authors: Alberto Alonso, Jasmine Camero, Alejandra Castillo, Fabrice O. Ulysse, Victoria Uribe, and Andrés R. Vindas Meléndez

\author{
Organizer Authors: Alexander \\ Diaz-Lopez, Pamela E. Harris, \\ Vanessa Rivera Quiñones, \\ Luis Sordo Vieira, Aris Winger, \\ and Michael Young
}

\section{Background}

The Mathematics Summer Workshop for Achieving Greater Graduate Educational Readiness (Math SWAGGERwww . mathswagger . com is a five-week virtual summer program for any underrepresented student (including women, underrepresented minorities-African Americans, American Indians including Native Alaskans, Latinxs/Hispanics

Alberto Alonso is a PhD student at Rochester Institute of Technology. His email address is a1berto1596@gmai1.com.

Jasmine Camero is a mathematics PhD student at Emory University. Her email address is jasmine.camero@emory.edu.

Alejandra Castillo is a PhD student in statistics at Oregon State University. Her email address is castiale@oregonstate.edu.

Fabrice O. Ulysse is a PhD student in the Department of Mathematics at the University of Notre Dame. His email address is fulysse@nd. edu.

Victoria Uribe is a PhD student in applied mathematics at Arizona State University. Her email address is vduribe@asu. edu.

Andrés R. Vindas Meléndez is a PhD candidate at the University of Kentucky. His email address is andres.vindas@uky.edu.

Alexander Diaz-Lopez is assistant professor of mathematics at Villanova University. His email address is a 1exander . di az-1opez@vi11 anova. edu.

Pamela E. Harris is associate professor of mathematics at Williams College. Her email address is peh2@wi11iams.edu.

Vanessa Rivera-Quiñones is a freelancer and instructor at the Sagrado Global Data Science \& Analytics program. Her email address is vriveraq .phd@gmai1 .com.

Luis Sordo Vieira is research assistant professor in the Division of Pulmonary, Critical Care and Sleep Medicine, Department of Medicine, University of Florida. His email address is 1uis . sordovi ei ra@medicine.uf1 . edu.

Aris Winger is assistant professor of mathematics at Georgia Gwinnett College. His email address is awinger@ggc.edu.

Michael Young is associate professor of mathematics at Iowa State University. His email address is myoung@iastate.edu.

DOI: https://dx.doi.org/10.1090/noti2183 
and Native Pacific Islanders-and persons with disabilities) who will be enrolled in a mathematical/statistical graduate program in Fall 2020. Through the workshop we discuss topics centered on the challenges faced by underrepresented students in those programs. The program is facilitated by a group of underrepresented mathematicians with broad experience in supporting student success.

\section{Building Community}

You often hear the words "mathematics community" used to refer to the general population of mathematicians. One could ask, "a community for whom?" It has been the experience of many underrepresented mathematicians that the larger "mathematics community" is not always welcoming, and can even be exclusionary at times. During a global pandemic, where social distancing is the new norm, and many academic communities are scattered beyond their institutions, the task of creating community in a space that is already difficult to navigate is a challenging one. This begs the question, can one actually recreate a community environment through such a virtual setting?

Math SWAGGER is a workshop whose mission is to provide strategies and community to incoming and current mathematics graduate students so that they too may thrive in their graduate studies and professional careers and it has provided evidence that community can in fact be recreated virtually. If you ask multiple people to define community, you will receive different answers. This is because everyone has different goals, visions, and desires. Math SWAGGER participants come searching for, and are very much in need of, a safe space to share their experiences and concerns pertaining to graduate school. The Math SWAGGER workshop provides these mathematicians with a space, and a community, in which they can share their experiences with other underrepresented mathematicians without worrying about being judged.

\section{People Above Mathematics}

This has been a phrase shared by several participants to explain why they do, and how they do, mathematics. The power and influence of people have kept many Math SWAGGER participants in the field. Do they love mathematics? YES. But for many of us, the support received, the friendships and mentorships formed, have been a driving force behind our continued involvement in the field. After all, it is people who help form a connection with the things we love doing. Without support, it may be challenging to continue to thrive by oneself. Although participating in SWAGGER means we do not meet in the same physical space, we are still open to sharing our experiences and learning from others. We cannot underestimate the power of storytelling. We are each the astonishing raconteurs of our own journeys. Everyone's experiences and stories are valuable. Emphasizing the telling and immediate support of participants' mathematical and personal stories can be an important first step toward recreating a successful virtual space like Math SWAGGER.

After all, we should all work to build "community" before adding any modifiers, such as "mathematics," before the word. By setting expectations for both the participants and the organizers and by also reflecting on what is required to build this space, we have constructed an academic community outside of our respective departments. The main component of our community is the requirement that participants be open and real. That is, participants are honest and open to having difficult conversations that might not take place in most (if any) academic spaces. The more we connect virtually, the more trust and vulnerability our community establishes. This leads to forming a stronger bond between individuals and allowing for the sharing of resources. Thus far, our difficult discussions have included topics such as community, self-care, motivation, and whiteness. It is through these difficult discussions, and framing these topics around our lived experiences, that we have grown as individuals and together as a community.

We propose the following items to consider when attempting to recreate a strong sense of community in virtual spaces:

1. Provide a space and ample time for sharing.

2. Commit to creating a welcoming environment that is enhanced by diverse experiences.

3. Respect everyone's ideas and experiences.

4. Listen actively. Listen to understand, rather than to reply.

5. Share as much as you're comfortable with-your voice matters! Also, speak from your own experience.

6. Be cognizant to include everyone in the conversation, even if it is a simple "What do you think?"

7. Give people credit, when possible, for the ideas or things they brought up: "[name] said this..." will make them feel heard and even more included in the conversation.

8. When in doubt, ask "Please say more about X?"

9. Be okay with silence. People need time to process, reflect, and gather ideas to share.

10. Break out into small groups of at most six people to facilitate conversation.

11. Have questions, activities, and scenarios ready to spark discussion.

\section{Workshop Logistics}

The Math SWAGGER application consisted of an online form where applicants shared their interests in participating in the program. In accepting the offer to join Math SWAGGER, participants signed an agreement to abide by community guidelines to help ensure a rewarding and welcoming experience for all.

Our Math SWAGGER community consists of seven organizers and thirty participants. Three times a week, participants and organizers meet on a virtual platform for an 
interactive ninety minute session. Everyone is encouraged to use their webcam, their microphone, and to participate through the platform's chat. Each session focuses on a topic such as: academic skills, family, self-care, just to name a few. These sessions are led by different organizers. This allows students to learn about unique aspects of each organizer's professional journey. The structure of each session varies, but a common format is to have an introduction to the topic for the first fifteen minutes, followed by breakout sessions lasting about forty-five minutes, and ending with a closing session.

Midway through the program, space was provided for participants to give formal feedback about the workshop. Throughout the entire program, however, participants are encouraged to share any concerns they may have about the structure of the program, what is helpful, and what is not.

Outside of the virtual meetings, each participant shares their ongoing reflections with organizers through a journal via a Google document. Prior to a meeting, participants prepare for a session by writing about their experience as it relates to the upcoming topic. After a meeting, organizers ask participants to discuss takeaways and provide action items they can commit to completing.

Communication has been very important to sustaining this program. Along with the virtual meetings, participants and organizers communicate through a Google group and Slack channel. These have also been venues for sharing materials, resources, and preparing for prelims.

We strongly believe that through careful organization and with a clear centering of people above mathematics, others can also recreate a community environment such as Math SWAGGER through a virtual setting.

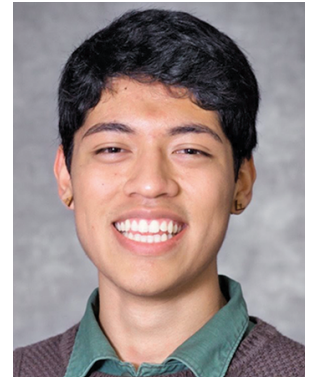

Alberto Alonso

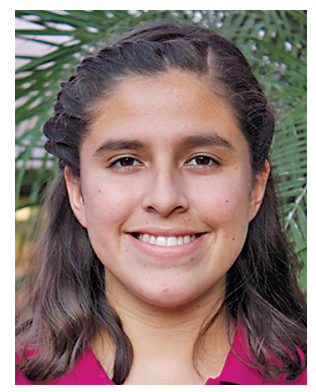

Alejandra Castillo

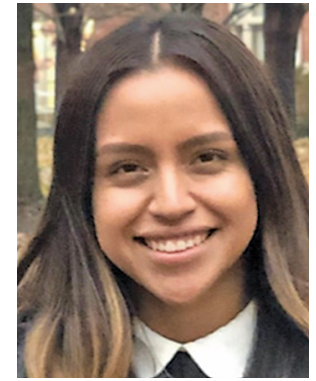

Jasmine Camero

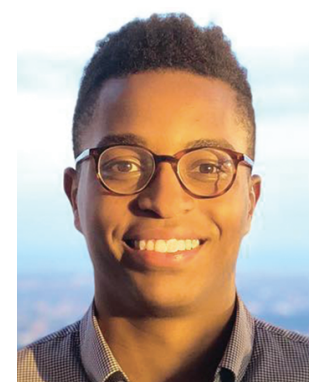

Fabrice O. Ulysse

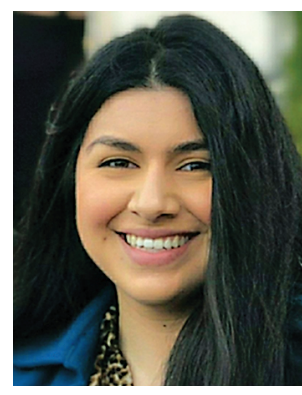

Victoria Uribe

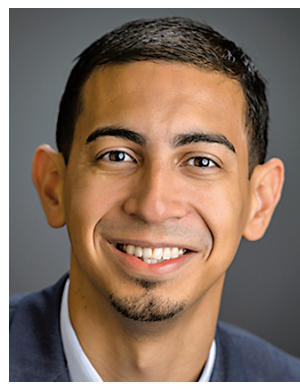

Alexander Diaz-Lopez

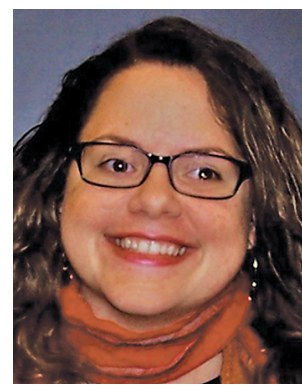

Vanessa Rivera

Quiñones

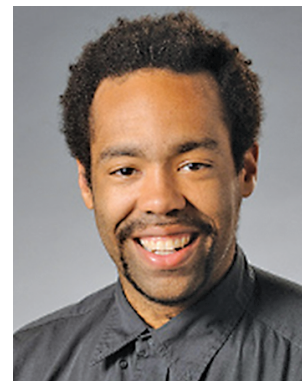

ArisWinger

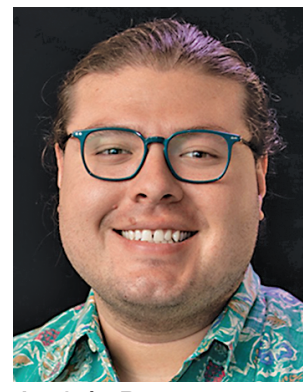

Andrés R.

Vindas Meléndez

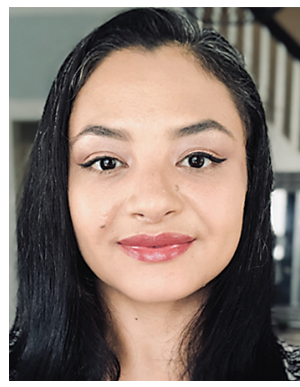

Pamela E. Harris

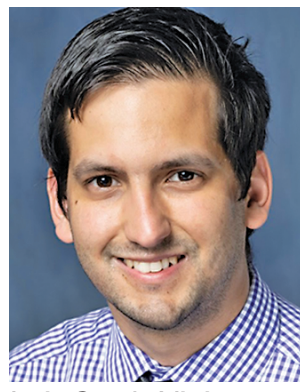

Luis Sordo Vieira

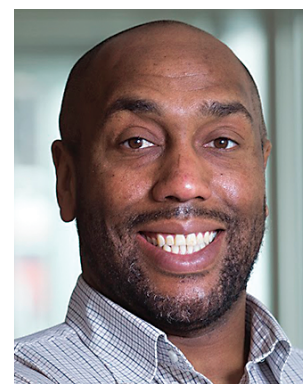

Michael Young
ACKNOWLEDGMENTS. The authors acknowledge funding support for Math SWAGGER through the National Science Foundation Award \#1744463.

\section{Credits}

Photo of Alberto Alonso is courtesy of Alberto Alonso. Photo of Jasmine Camero is by Roberto Hernandez. Photo of Alejandra Castillo is courtesy of Alejandra Castillo. Photo of Fabrice O. Ulysse is courtesy of Marc Antonios. Photo of Victoria Uribe is courtesy of Victoria Uribe. 
Photo of Andrés Vindas Meléndez is courtesy of Hive (Creative \& Technical Services), College of Arts \& Sciences, University of Kentucky.

Photo of Alexander Diaz-Lopez is courtesy of Villanova University.

Photo of Pamela Harris is courtesy of Pamela Harris.

Photo of Vanessa Rivera Quiñones is courtesy of the Department of Mathematics, University of Illinois at Urbana-Champaign.

Photo of Luis Sordo Vieira is courtesy of University of Florida.

Photo of Aris Winger is courtesy of Georgia Gwinett College. Photo of Michael Young is courtesy of Iowa State University.

\section{Organize a Laid-Back Conference in the Rocky Mountains that Participants Want to Return to Year after Year}

\section{Danny Krashen and Kelly McKinnie}

We are at a difficult moment presently as a community, where many aspects of our professional life are rapidly changing due to the global pandemic. In particular, the structure of conferences and seminars has been in a state of flux, as new models are being explored and in-person meetings are being canceled.

In this context, it may be useful to take a step back and try and assess our community goals. Why is it that we organize and attend conferences? What do we hope to get out of them, and what compels us to organize them? Who typically benefits most from them? When the world returns to a place where we can once again organize in-person conferences, what will we have learned? What differences should we see in future conferences?

In fact, there is probably a diverse set of valid answers to these questions. It is not our intention here to weigh the pros and cons of various choices, but rather to explore one particular set of choices, which the authors' small community has made, as the larger mathematical community moves in a new direction for conference organization.

The original conception of the "Brauer group" meetings was simple: to establish a community of mathematicians, loosely interested in the same math, who enjoy spending time together in the mountains. Implicit in this

Danny Krashen is professor of mathematics at Rutgers University. His email address is danie1.krashen@rutgers. edu.

Kelly McKinnie is associate professor of mathematics at the University of Montana. Her email address is ke11y.mckinnie@mso. umt. edu.

DOI: https://dx.doi.org/10.1090/noti 2181 organization though was the idea that spending unstructured time together, appreciating the Rocky Mountains, would be a great way to form long-lasting professional and personal connections. In addition, the accepting and generous behavior of senior participants at the beginnings of these meetings had a huge role in setting the tone for norms and expectations of behavior for years to come. Together, these were very effective in building a cohesive and supportive professional community of researchers.

In this article, we'll first talk a bit about the organic beginnings of the Brauer group meetings, and its particular features which we have found helpful in shaping our community. We'll then say a bit about how we've used these ideas at other related conferences, and end with some thoughts about the future.

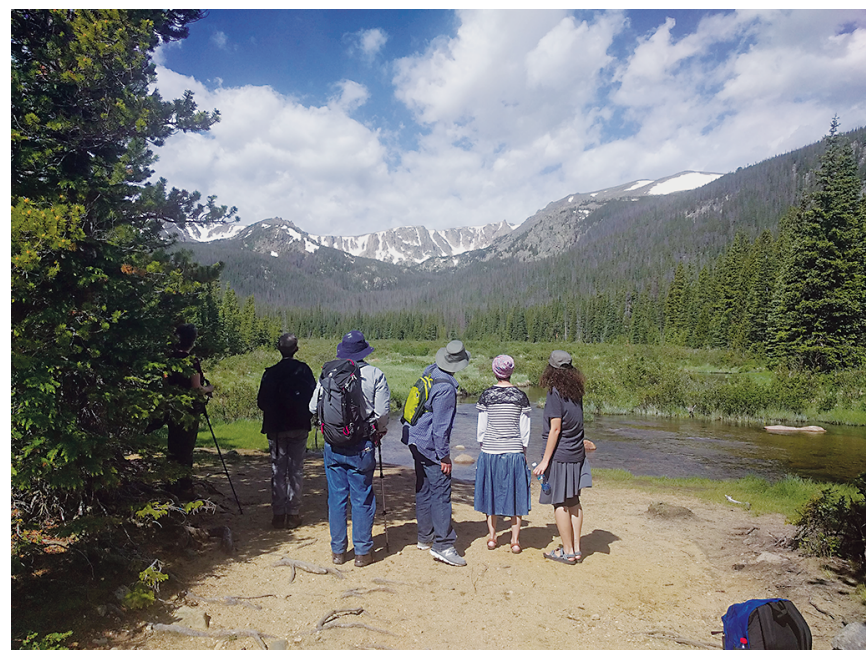

Figure 1.

Folklore has it that the Brauer group meetings began over 30 years ago when David Saltman and Darrell Haile, both algebraists who liked to hike, met up with Frank DeMeyer at Colorado State University. They drove up a mountain to start a hike and came upon the Mountain Branch Campus. This is and was a primitive "campus" designed to house and feed forestry students from CSU. It has some dorm and private room accommodations, a lovely old classroom building with a tiny chalkboard and a cozy fireplace with beautiful views of the mountain valley from the deck. One of the group said something to the effect of "We should host a conference here so we can hike a lot," and voila, the Brauer group meetings were born.

We, the authors, first started attending the conferences around 2000, about 10 years after their beginnings. At this time, both of us were students of David Saltman, and attending them was a highlight of our graduate school years. Since around 2008, we have been a part of the organizing committee for this conference. It has been held about every two to three years over the past 30 years with a few meetings 